OPEN ACCESS

Edited by:

Smaranda Leu-Semenescu, Pitie-Salpetriere Hospital, France

Reviewed by:

Takashi Kanbayashi,

University of Tsukuba, Japan

J. M. Monti,

University of the Republic, Uruguay

${ }^{*}$ Correspondence:

Yong Won Cho

neurocho@gmail.com

${ }^{\dagger}$ Deceased

Specialty section:

This article was submitted to

Sleep Disorders,

a section of the journal

Frontiers in Neurology

Received: 30 September 2021

Accepted: 20 October 2021

Published: 29 November 2021

Citation:

Bae H, Cho YW, Kim KT, Allen RP and

Earley CJ (2021) The Safety and

Efficacy of Pregabalin Add-on Therapy

in Restless Legs Syndrome Patients.

Front. Neurol. 12:786408

doi: 10.3389/fneur.2021.786408

\section{The Safety and Efficacy of Pregabalin Add-on Therapy in Restless Legs Syndrome Patients}

\author{
Hyoeun Bae ${ }^{1}$, Yong Won Cho ${ }^{1 *}$, Keun Tae Kim ${ }^{1}$, Richard P. Allen ${ }^{2 \dagger}$ and \\ Christopher J. Earley ${ }^{2}$
}

${ }^{1}$ Department of Neurology, Keimyung University School of Medicine, Daegu, South Korea, ${ }^{2}$ Department of Neurology, Hopkins Bayview Medical Center, Johns Hopkins University, Baltimore, MD, United States

Pregabalin is increasingly being used as a first-line treatment for symptomatic control of restless legs syndrome (RLS). This study aimed to evaluate the efficacy and safety of pregabalin as add-on therapy in RLS patients already taking dopamine agonists (DA) but still in need of further management. Patients with idiopathic RLS were enrolled, and all had already been prescribed DA for at least 3 months but still had either persistent symptoms, side effects, or comorbid insomnia. An initial dose of $75 \mathrm{mg}$ pregabalin was begun, adjusted as needed, and maintained at a stable dose for 4 weeks, followed by observation for a total of 8 weeks. RLS symptoms and insomnia scores were evaluated before and after add-on pregabalin treatment. Patients were monitored for side effects that could be attributed to pregabalin. A total of 32 RLS patients were enrolled, and 20 subjects remained until the endpoint. After the pregabalin add-on, the mean IRLS score showed significant improvement compared to the baseline $(p<0.001)$. The insomnia severity index score also improved $(p=0.036)$, and no serious adverse effects were observed. Our preliminary data suggests the potential for pregabalin as an add-on therapy to DA with regards to both efficacy and safety in patients who have inadequate RLS improvement.

Keywords: restless legs syndrome, pregabalin, dopamine agonists, treatment, add-on

\section{INTRODUCTION}

Restless legs syndrome is a sensorimotor neurological disorder with a worldwide prevalence of $5-10 \%$, and $2-3 \%$ of patients having symptoms severe enough to seek treatment (1). The discomfort and urge to move the legs, which is the prominent symptom of restless legs syndrome (RLS), occurs mainly at night. Symptomatic patients suffer from sleep disturbance and their quality of life deteriorates as a consequence (2).

Dopamine agonists (DA) are known to relieve RLS symptoms (3) and are commonly used as first-line treatment (4). Acute treatment with DA can result in side effects that may limit dose escalation and thus limit the ability of DA to fully treat the symptoms. Adverse events were documented for $79 \%$ during the first trial year of rotigotine (5), and $19-24 \%$ discontinued pramipexole by 1 year (6). Previous studies have demonstrated that despite improving symptoms, DA does not always improve sleep quality (7) and it may cause insomnia in some patients. Finally, some patients may only have partial benefits even at the acceptable, upper-dose limits. Treatment options for physicians who have to deal with these problems when trying to treat patients include 
moving to an alternative class of agents or add-on a second medication from an alternative class of agents. One of these alternative agents is pregabalin (6).

Pregabalin has been shown to be an effective monotherapy for managing RLS symptoms when compared to placebo or active treatment with a DA $(6,8-10)$. However, there have been no studies using pregabalin as an add-on agent with DA. Studies with pregabalin in RLS have reported that pregabalin is effective in improving not only RLS symptoms but also sleep structure $(6,9)$. Other drugs are considered second-line treatment options and have several side effects. Benzodiazepines have dependency, falling, and reduction of deep sleep issues. Opioids have many side effects such as sedation, constipation, mood change, opioidinduced respiratory depression, and dependence (11). Therefore, pregabalin shows promise as a non-dopaminergic "add-on" treatment option that might complement and compensate for some limitations with the use of single-agent DA $(4,12)$. The purpose of this study was to evaluate the efficacy and safety of pregabalin as add-on therapy in patients who were on DA for RLS but for whom further symptomatic improvement was needed.

\section{METHODS}

\section{Study Design and Participants}

The study was approved by the institutional Ethics Committee of the regional hospital with written informed consent obtained from all participants. It was a prospective single-center study, conducted between January 2019 and December 2020. All patients were initially on a daily regime of DA for at least 3 months and needed additional treatment due to comorbid insomnia or persistence of symptoms despite reaching upper limits of DA dose or dose escalation was limited due to onset of side-effects.

Patients aged over 18 with idiopathic RLS symptoms were eligible for the study. RLS was diagnosed by a neurologist through a face-to-face interview based on a Korean version of the Hopkins Telephone Diagnostic Interview. Patients were required to be taking a stable dose of a DA for at least 3 months and no other RLS treatments. Enrollees in the study were required to have symptoms on at least 5 days per week or at least have 2 days of International RLS (IRLS rating scale) score over 15 per week. We evaluated for augmentation using the National Institutes of Health (NIH) criteria for augmentation (13).

The exclusion criteria were as follows: secondary RLS (e.g., peripheral neuropathy, neurodegenerative disorders, multiple sclerosis, and chronic renal failure); the presence of DA-induced augmentation; receiving any treatment that can markedly change RLS symptoms or study results, including anxiolytics or antidepressants; severe medical illness (e.g., chronic organ failure, active inflammation or infection, and congestive heart failure); any condition that causes iron deficiency (e.g., pregnancy, chronic bleeding, excluding menstruation and medically necessary phlebotomy); shift worker; untreated obstructive sleep apnea; prior experience of adverse effects to pregabalin; inability to participate in the trial; and history of suicidal thoughts or attempts within 6 months.

Baseline evaluations were conducted at the first visit. Demographic data, iron profiles, sleep questionnaires including
Pittsburg sleep quality index (PSQI) (14), Insomnia severity index (ISI) (15), Beck Depression Inventory (BDI) (16), Beck Anxiety Inventory (BAI) (17), and evaluation of RLS symptoms by IRLS (18), RLS quality of life (RLS QoL) (19), Visual Analog Scale (VAS) were assessed. The questionnaires were validated in a Korean version. The initial dose of pregabalin was a single $75 \mathrm{mg}$ dose in the evening, and it was adjusted up to $300 \mathrm{mg}$ over 4 weeks depending on the symptoms of the patient.

Afterward, 4 weeks from the first visit, the patient was evaluated for changes in RLS symptoms and side effects of the pregabalin, and to determine a final and stable dose for the following 4 weeks.

The final visit was 8 weeks from the first visit. Again, symptom changes and adverse effects, including augmentation, were checked. In addition, the same evaluation conducted at the first visit (i.e., iron profiles, sleep scales, and RLS symptom evaluation) were repeated.

\section{Statistical Analysis}

Clinical information, RLS symptom, and sleep status were analyzed by frequency analysis and descriptive statistics. Changes in RLS symptoms and sleep status after pregabalin treatment were analyzed using paired $t$-test. The primary variables were IRLS score and VAS. The analyses were based on an intentionto-treat model with mean substitution of imputation technique. The Pearson's correlation and multiple regression were used for the analysis of the relationship between clinical information.

\section{RESULTS}

A total of 32 participants were enrolled at the first visit: six had side effects limiting increases in DA, 10 had co-morbid insomnia, and 16 had persistence of symptoms. Among the 32 participants, 12 had dropped out by Week 8 . The causes of the dropouts were as follows: three were related to drug adverse effects (dizziness or sedation), two were unwilling to travel the long distance required, three were related to drug ineffectiveness, and four had personal reasons for not returning to the clinic, but unrelated to drug issues. In this group, the dose of pramipexole $(N=8)$ ranged from 0.125 to $0.75 \mathrm{mg}$ and ropinirole $(N=4)$ from 0.25 to $2 \mathrm{mg}$. The median (range) treatment duration with DA was $727.08 \pm 867.21$ days (91-2,529 days). Additionally, 20 participants completed the Week 8 endpoint (Figure 1). The dose of pramipexole $(N=18)$ ranged from 0.125 to $0.5 \mathrm{mg}$, and ropinirole $(N=2)$ from 0.75 to $2 \mathrm{mg}$. The median (range) treatment duration with DA of the 20 participants was 472.65 \pm 427.45 days (92-2,376 days). At Week 8 endpoint, 19 patients were on pregabalin $75 \mathrm{mg}$ and one was on pregabalin $150 \mathrm{mg}$.

Regarding further results, 81 percent were women, mean age $59 \pm 13$ years. RLS duration was $12 \pm 10$ years with IRLS 25.7 \pm 7.2 , and RLS VAS $51.9 \pm 32.9$ when they enrolled. Moreover, 7 (21.9\%) patients had clinical insomnia with ISI over 15, 15 (46.9\%) were poor sleepers with PSQI over 8.5, 2 (6.3\%) showed anxiety, while 10 (31.3\%) had depressed moods. Hemoglobin and ferritin were within the normal range.

After adding pregabalin, RLS severity was significantly improved with mean IRLS lowered from 26.5 to $20.9(p<0.001)$ and the ISI showed statistically significant improvement 


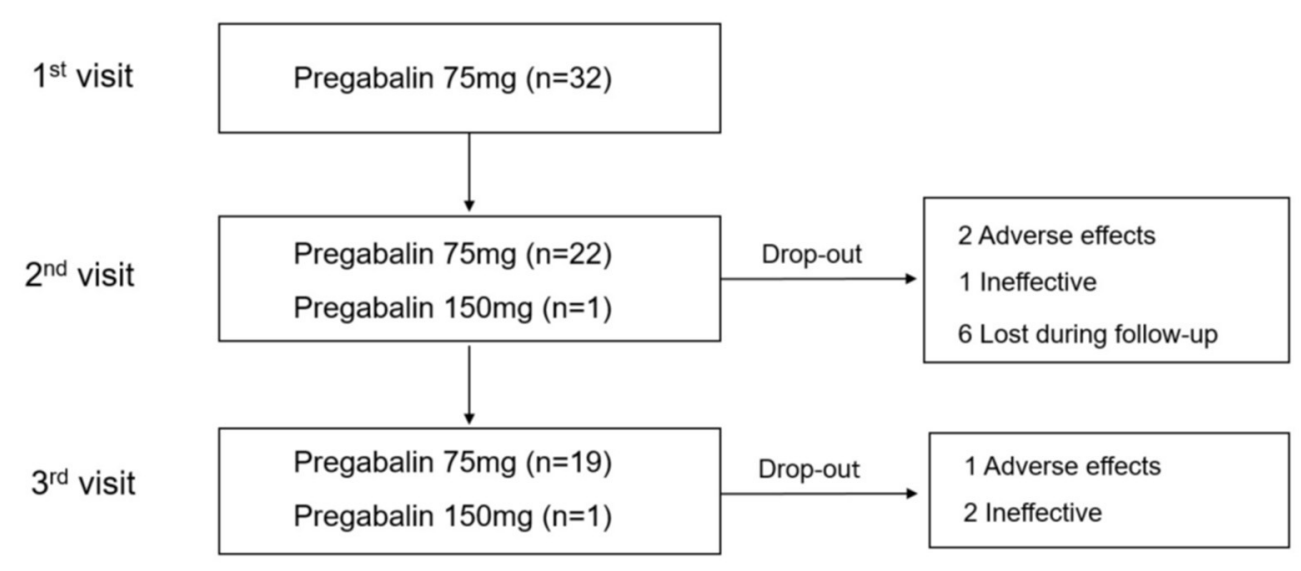

FIGURE 1 | Study flow chart.

TABLE 1 | Mean ( \pm SD) for the pre- and post-treatment RLS severity and sleep scale score.

\begin{tabular}{lccc}
\hline & At baseline & Post-treatment & $\boldsymbol{p}$ \\
\hline IRLS & $26.48 \pm 8.10$ & $20.91 \pm 8.66$ & $<0.001$ \\
RLS Qol & $68.63 \pm 20.58$ & $69.50 \pm 22.38$ & 0.068 \\
RLS VAS & $57.14 \pm 34.81$ & $42.38 \pm 29.82$ & 0.139 \\
ISI & $13.60 \pm 7.65$ & $12.65 \pm 7.97$ & 0.036 \\
PSQI & $11.24 \pm 4.35$ & $11.00 \pm 4.59$ & 0.093 \\
BAI & $11.10 \pm 9.48$ & $10.62 \pm 9.63$ & 0.431 \\
BDI & $13.05 \pm 9.94$ & $13.57 \pm 9.53$ & 0.330
\end{tabular}

$R L S$, Restless legs syndrome; IRLS, International Restless Legs Scale; RLS Qol, RLS specific quality of life questionnaire; VAS, visual analog scale of severity; ISI, Insomnia Severity Index; PSQI, Pittsburg sleep quality index; BDI, Beck Depression Inventory; BAI, Beck Anxiety Inventory.

(Table 1). None of the other scales were significantly different from the baseline. A total of six patients reported the following adverse effects on pregabalin: dizziness in two patients; sleepiness in two patients, drooling in one patient, and mild rash in one patient. Among the six patients who reported side effects, three completed the Week 8 visit.

\section{DISCUSSION}

This study showed the usefulness of pregabalin as an add-on treatment in patients who continue to have problems with RLS, despite DA use, over 8 weeks. From the perspective of RLS severity, adding pregabalin resulted in a statistically significant improvement, manifested by an improved IRLS score.

Pregabalin binds to voltage-gated calcium channels, inhibits calcium entry, and results in lowering glutamate activity and therefore decreasing excitation (20). This mechanism is hypothesized to exert an effect on RLS treatment (12). Pregabalin has several additional beneficial aspects in the management of RLS that should also be considered. Firstly, pregabalin has a positive effect on sleep (21). Current guidelines for RLS management also suggest that if patients have comorbid insomnia or sleep disturbance disproportionate to other symptoms of RLS, alpha-2-delta ligands could be first-line treatment (4). In our study, ISI was improved, indicating that pregabalin could be helpful as an add-on therapy for RLS patients with insomnia. Secondly, pregabalin is capable of controlling other comorbidities in RLS patients. As one of the alpha-2delta ligands, it can be effective in controlling neuropathic pains and anxiety, for example (12). In addition, interaction with the BK channel has been suggested to reduce neuropathic pains (22). As regards its safety, pregabalin was well-tolerated, in line with previous studies that reported pregabalin is generally welltolerated (9).

Generally, pregabalin is prescribed at doses ranging from 50 to $450 \mathrm{mg}$ (4) and previous randomized controlled trials showed an effect in doses of over $150 \mathrm{mg}$ as monotherapy $(6,9,10)$. In our study, the dose of $75 \mathrm{mg}$ was maintained in the majority of patients as add-on treatment. This relatively low dosage may be related to the fact that pregabalin was used as add-on therapy, not as monotherapy where dosages are routinely higher.

This study has several limitations. Our sample size was small, and the drop-out rate was moderate. The study was an unblinded, open-label study for which placebo effects could contribute to the effects we measured. Measures of sleep quality and periodic legs movement quantification during sleep on overnight polysomnography would have provided more "objective" treatment measurements.

In patients who continued to have problems with RLS symptoms despite being on a DA, pregabalin might be considered as add-on therapy to further improve RLS symptoms, thereby reducing some of the covariant insomnia problems associated with DA usage. The data supported the value of a future randomized, double-blind, placebo-controlled clinical trial in a larger population of RLS patients for whom DA are primary treatments but are not fully effective.

\section{DATA AVAILABILITY STATEMENT}

The original contributions presented in the study are included in the article/supplementary files, further inquiries can be directed to the corresponding author. 


\section{ETHICS STATEMENT}

The studies involving human participants were reviewed and approved by Keimyung University Dongsan Medical Center. The patients/participants provided their written informed consent to participate in this study.

\section{AUTHOR CONTRIBUTIONS}

YC and CE conceptualized research. $\mathrm{HB}$ and YC made contributions to the investigation. $\mathrm{KK}, \mathrm{YC}$, and $\mathrm{CE}$ analyzed

\section{REFERENCES}

1. Allen RP, Walters AS, Montplaisir J, Hening W, Myers A, Bell TJ, et al. Restless legs syndrome prevalence and impact: REST general population study. Arch Intern Med. (2005) 165:1286-92. doi: 10.1001/archinte.165.11.1286

2. Winkelman JW, Redline S, Baldwin CM, Resnick HE, Newman AB, Gottlieb DJ. Polysomnographic and health-related quality of life correlates of restless legs syndrome in the Sleep Heart Health Study. Sleep. (2009) 32:7728. doi: $10.1093 /$ sleep/32.6.772

3. Trenkwalder C, Hening WA, Montagna P, Oertel WH, Allen RP, Walters AS, et al. Treatment of restless legs syndrome: an evidence-based review and implications for clinical practice. Mov Disord. (2008) 23:2267302. doi: $10.1002 / \mathrm{mds} .22254$

4. Garcia-Borreguero D, Silber MH, Winkelman JW, Hogl B, Bainbridge J, Buchfuhrer M, et al. Guidelines for the first-line treatment of restless legs syndrome/Willis-Ekbom disease, prevention and treatment of dopaminergic augmentation: a combined task force of the IRLSSG, EURLSSG, and the RLS-foundation. Sleep Med. (2016) 21:1-11. doi: 10.1016/j.sleep.2016.01.017

5. Oertel WH, Benes H, Garcia-Borreguero D, Geisler P, Högl B, Trenkwalder C, et al. One year open-label safety and efficacy trial with rotigotine transdermal patch in moderate to severe idiopathic restless legs syndrome. Sleep Med. (2008) 9:865-73. doi: 10.1016/j.sleep.2008.04.012

6. Allen RP, Chen C, Garcia-Borreguero D, Polo O, DuBrava S, Miceli J, et al. Comparison of pregabalin with pramipexole for restless legs syndrome. $\mathrm{N}$ Engl J Med. (2014) 370:621-31. doi: 10.1056/NEJMoa1303646

7. Partinen M, Hirvonen K, Jama L, Alakuijala A, Hublin C, Tamminen I, et al. Efficacy and safety of pramipexole in idiopathic restless legs syndrome: a polysomnographic dose-finding study-the PRELUDE study. Sleep Med. (2006) 7:407-17. doi: 10.1016/j.sleep.2006.03.011

8. Garcia-Borreguero D, Patrick J, DuBrava S, Becker PM, Lankford A, Chen C, et al. Pregabalin versus pramipexole: effects on sleep disturbance in restless legs syndrome. Sleep. (2014) 37:635-43. doi: 10.5665/sleep.3558

9. Allen R, Chen C, Soaita A, Wohlberg C, Knapp L, Peterson BT, et al. A randomized, double-blind, 6-week, dose-ranging study of pregabalin in patients with restless legs syndrome. Sleep Med. (2010) 11:5129. doi: 10.1016/j.sleep.2010.03.003

10. Garcia-Borreguero D, Larrosa O, Williams A-M, Albares J, Pascual M, Palacios J, et al. Treatment of restless legs syndrome with pregabalin: a double-blind, placebo-controlled study. Neurology. (2010) 74:1897904. doi: 10.1212/WNL.0b013e3181e1ce73

11. Wanner V, Malo CG, Romero S, Cano-Pumarega I, GarcíaBorreguero D. Non-dopaminergic vs. dopaminergic treatment options in restless legs syndrome. Adv Pharmacol. (2019) 84:187-205. doi: 10.1016/bs.apha.2019.02.003

12. Faulkner MA. Use of $\alpha 2 \delta$ ligands for restless legs syndrome/Willis Ekbom disease. CNS Drugs. (2018) 32:149-59. doi: 10.1007/s40263-018-0502-z

13. Allen RP, Picchietti D, Hening WA, Trenkwalder C, Walters AS, Montplaisi J. Restless legs syndrome: diagnostic criteria, special considerations, and epidemiology: a report from the restless legs syndrome diagnosis and epidemiology workshop at the National Institutes of Health. Sleep Med. (2003) 4:101-19. doi: 10.1016/S1389-9457(03)00010-8 data. HB wrote the original draft. YC, CE, and RA supervised. All authors have reviewed and edited and contributed to data curation and methodology.

\section{FUNDING}

This study was supported by funding from Pharmascience Korea, Inc. Pharmascience Korea was not involved in the study design, collection, analysis, interpretation of data, the writing of this article, or the decision to submit it for publication.

14. Sohn SI, Kim DH, Lee MY, Cho YW. The reliability and validity of the Korean version of the Pittsburgh Sleep Quality Index. Sleep Breath. (2012) 16:803-12. doi: 10.1007/s11325-011-0579-9

15. Cho YW, Song ML, Morin CM. Validation of a Korean version of the insomnia severity index. J Clin Neurol. (2014) 10:210-5. doi: $10.3988 /$ jen.2014.10.3.210

16. Lim S-Y, Lee E-J, Jeong S-W, Kim H-C, Jeong C-H, Jeon T-Y, et al. The validation study of Beck Depression Scale 2 in Korean version. Anxiety Mood. (2011) 7:48-53.

17. Yook S, Kim Z. A clinical study on the Korean version of Beck Anxiety Inventory: comparative study of patient and non-patient. Korean J Clin Psychol. (1997) 16:185-97.

18. Yang J-G, Kim D-H, Lee J-H, Park K-H, Jung K-Y, Shin W-C, et al. The reliability and validity of the Korean versions of the international restless legs scale and the restless legs syndrome quality of life questionnaire. Journal of the Korean neurological association. (2010) 28:263-9.

19. Abetz L, Vallow SM, Kirsch J, Allen RP, Washburn T, Earley CJ. Validation of the restless legs syndrome quality of life questionnaire. Value in Health. (2005) 8:157-67. doi: 10.1111/j.1524-4733.2005.03010.x

20. Fink K, Dooley DJ, Meder WP, Suman-Chauhan N, Duffy S, Clusmann $\mathrm{H}$, et al. Inhibition of neuronal $\mathrm{Ca} 2+$ influx by gabapentin and pregabalin in the human neocortex. Neuropharmacology. (2002) 42:22936. doi: 10.1016/S0028-3908(01)00172-1

21. Russell IJ, Crofford LJ, Leon T, Cappelleri JC, Bushmakin AG, Whalen $E$, et al. The effects of pregabalin on sleep disturbance symptoms among individuals with fibromyalgia syndrome. Sleep Med. (2009) 10:60410. doi: 10.1016/j.sleep.2009.01.009

22. Zhang F-X, Gadotti VM, Souza IA, Chen L, Zamponi GW, BK. potassium channels suppress Cav $\alpha 2 \delta$ subunit function to reduce inflammatory and neuropathic pain. Cell Rep. (2018) 22:1956-64. doi: 10.1016/j.celrep.2018. 01.073

Conflict of Interest: This study received funding from Pharmascience Korea, Inc. The funder was not involved in the study design, collection, analysis, interpretation of data, the writing of this article or the decision to submit it for publication. All authors declare no other competing interests.

Publisher's Note: All claims expressed in this article are solely those of the authors and do not necessarily represent those of their affiliated organizations, or those of the publisher, the editors and the reviewers. Any product that may be evaluated in this article, or claim that may be made by its manufacturer, is not guaranteed or endorsed by the publisher.

Copyright $\odot 2021$ Bae, Cho, Kim, Allen and Earley. This is an open-access article distributed under the terms of the Creative Commons Attribution License (CC BY). The use, distribution or reproduction in other forums is permitted, provided the original author(s) and the copyright owner(s) are credited and that the original publication in this journal is cited, in accordance with accepted academic practice. No use, distribution or reproduction is permitted which does not comply with these terms. 